\title{
Musical training intensity yields opposite effects on grey matter density in cognitive versus sensorimotor networks
}

\author{
Clara E. James • Mathias S. Oechslin • \\ Dimitri Van De Ville $\cdot$ Claude-Alain Hauert • \\ Céline Descloux $\cdot$ François Lazeyras
}

Received: 26 July 2012/ Accepted: 17 December 2012

(c) Springer-Verlag Berlin Heidelberg 2013

\begin{abstract}
Using optimized voxel-based morphometry, we performed grey matter density analyses on 59 age-, sexand intelligence-matched young adults with three distinct, progressive levels of musical training intensity or expertise. Structural brain adaptations in musicians have been repeatedly demonstrated in areas involved in auditory perception and motor skills. However, musical activities are not confined to auditory perception and motor performance, but are entangled with higher-order cognitive processes. In consequence, neuronal systems involved in such higher-order processing may also be shaped by experience-
\end{abstract}

Electronic supplementary material The online version of this article (doi:10.1007/s00429-013-0504-z) contains supplementary material, which is available to authorized users.

C. E. James - M. S. Oechslin · D. Van De Ville · C.-A. Hauert Geneva Neuroscience Center, University of Geneva,

Geneva, Switzerland

C. E. James $(\varangle) \cdot$ M. S. Oechslin · C.-A. Hauert

Faculty of Psychology and Educational Sciences,

University of Geneva, Uni Mail, 40 Bd Du Pont d'Arve,

1205 Geneva, Switzerland

e-mail: clara.james@unige.ch

C. E. James

University of Applied Sciences of Western Switzerland,

Health, Geneva, Switzerland

D. Van De Ville · F. Lazeyras

Faculty of Medicine, Department of Radiology and Medical

Informatics, University of Geneva, Geneva, Switzerland

D. Van De Ville

Institute of Bioengineering, École Polytechnique Fédérale

de Lausanne, Lausanne, Switzerland

C. Descloux

Medical University of Lausanne, Lausanne, Switzerland driven plasticity. We modelled expertise as a three-level regressor to study possible linear relationships of expertise with grey matter density. The key finding of this study resides in a functional dissimilarity between areas exhibiting increase versus decrease of grey matter as a function of musical expertise. Grey matter density increased with expertise in areas known for their involvement in higherorder cognitive processing: right fusiform gyrus (visual pattern recognition), right mid orbital gyrus (tonal sensitivity), left inferior frontal gyrus (syntactic processing, executive function, working memory), left intraparietal sulcus (visuo-motor coordination) and bilateral posterior cerebellar Crus II (executive function, working memory) and in auditory processing: left Heschl's gyrus. Conversely, grey matter density decreased with expertise in bilateral perirolandic and striatal areas that are related to sensorimotor function, possibly reflecting high automation of motor skills. Moreover, a multiple regression analysis evidenced that grey matter density in the right mid orbital area and the inferior frontal gyrus predicted accuracy in detecting fine-grained incongruities in tonal music.

Keywords Musical training - Voxel-based morphometry · Grey matter density · Plasticity · Cognition · Sensorimotor function

\section{Introduction}

Plasticity of the brain, its ability to structurally and functionally adapt in response to environmental demands, relies importantly on the nature and intensity of its use (Blakemore and Frith 2005).

Musical expertise is a rising star amongst topics investigating experience-driven brain plasticity (Pantev and 
Herholz 2011; Wan and Schlaug 2010; Jancke 2009) for two reasons. First because mastering a musical instrument fosters a panoply of intricately linked sensorimotor (Zatorre et al. 2007; Schneider et al. 2002) and higherorder cognitive functions (Oechslin et al. 2012; Schulze et al. 2011; Sluming et al. 2007). Second because musical expertise levels vary continuously from people solely exposed passively to musical stimuli in their environment, to highly trained expert performers.

The latter aspect has not been investigated extensively in neuroscience research on musicians' brain plasticity. Hence, the major interest of the present study resides in the fact that we studied whole-head grey matter differences as a function of three strictly controlled, distinct and progressive levels of musical expertise. In contrast to musicians versus non-musicians comparisons, including an intermediate level of proficiency allows identifying brain regions that are susceptible to progressive experience-driven neural adaptations.

Grey matter structural differences between the brains of musicians and non-musicians are known to manifest inter alia in the cerebellum (Hutchinson et al. 2003; Schlaug et al. 1995), primary auditory cortex (Schneider et al. 2002, 2005; Bermudez and Zatorre 2005), and primary and associative motor areas (Gaser and Schlaug 2003; Schlaug 2001; Hyde et al. 2009). Such structural adaptations appear to be intimately related to functional adaptations (Schneider et al. 2002; Zatorre et al. 2012).

As already stated, musical perception and execution are not limited to auditory processing and sensorimotor function, but also involve higher-order cognitive, mnemonic and attentional mechanisms (Janata et al. 2002b; Oechslin et al. 2012). Processing of musical syntax requires profound knowledge and comprehension of musical structure as well as keeping track of the short- and long-term musical context (Oechslin et al. 2012). Expert musicians encode, manipulate, and retrieve information differently compared to nonexperts (Williamon et al. 2002). Musicians demonstrated faster updating of auditory and visual working memory representations compared to non-musicians (George and Coch 2011). Instrumental performance also relies strongly on attentional focus (Janata et al. 2002b; Williamon et al. 2002). Therefore, experience-driven brain plasticity may not only modulate central auditory and motor processing but also neuronal systems involved in higher-order processing. Advantages with respect to higher-order cognitive functioning as a consequence of musical expertise have been shown previously for verbal working memory (Chan et al. 1998), executive control (Bialystok and Depape 2009), linguistic perception abilities (Moreno et al. 2009) and spatio-temporal reasoning (Rauscher et al. 1997).

Structural neuroplasticity seems to follow the law "more skill, more grey matter" (Gaser and Schlaug 2003; Sluming et al. 2002; Draganski et al. 2004; Hyde et al. 2009). However, new evidence exists that training and ensuing expertise sometimes induce local decrease of cortical volume (Granert et al. 2011; Hanggi et al. 2010). Skill acquisition provokes synaptic enhancement in frequently used connections that are strengthened whereas infrequently used connections are eliminated through pruning (Kanai and Rees 2011; Zatorre et al. 2012). In ballet dancers (Hanggi et al. 2010) as well as skilled pianists (Granert et al. 2011), striatal volume decreased with increasing motor function efficiency.

Given this background, we searched here for both positive and negative structural neural correlates reflecting stepwise increase in musical expertise. We performed regression analyses on whole-head grey matter density as a function of musical training intensity in three groups of young adults (non-musicians, amateur and professional "expert" pianists) matched for sex, age, age of training onset and fluid intelligence. We could verify the progressive increase in musical expertise of the groups by means of behavioural data from a recent functional MRI study (Oechslin et al. 2012) in which the same groups of subjects participated. The in-scanner task consisted in detection of refined multi-level musical transgressions.

According to the above-mentioned findings, we expected to observe progressive changes in grey matter density as a consequence of musical training in auditory and motor function related brain areas as well as in regions hosting universal functions of working memory and attention. We merely anticipated increases, but expected to possibly replicate findings reporting reduction of grey matter density with increasing musical skill in striatal areas. Because both musician groups were classically schooled pianists, we also anticipated some instrument-specific adaptations.

We could also establish relationships between grey matter density data from the current study, and behavioural in-scanner and fMRI results from the same group of participants (Oechslin et al. 2012). Zatorre et al. (2012) recently emphasized that brain function and structure are dynamically linked and advocated that searching for network-level patterns in anatomical structures is plausible. We hypothesized that grey matter density in areas involved in higher-order cognitive processing may predict behavioural results of music syntactic processing.

\section{Methods}

Participants

Fifty-nine volunteers gave written informed consent to take part in this experiment and received financial compensation. All participants were right-handed according to the 
Edinburgh Inventory (Oldfield 1971), reported normal hearing and presented no history of neurological illnesses. The protocol was approved by the local ethics committee.

Groups consisted of 20 professional pianists (experts; $24.5 \pm 4.5$ years; 10 women), 20 amateur pianists (amateurs; $22.2 \pm 3.1$ years; 11 women) and 19 non-musicians (non-musicians; $24.0 \pm 4.5$ years; 9 women). The groups were matched for gender and age (one-way ANOVA on age $F_{2,56}=2.2, p=0.12$ ). No significant group differences existed for fluid intelligence (Raven's Advanced Progressive Matrices Set II; Raven et al. 2003; $F_{2,58}=1.2$, $p=0.31$ ). One supplementary non-musician was removed from the analysis, because of a data acquisition artefact. Experts were mainly advanced conservatory students but also established artists or teachers, who received their training at the Conservatoires of Geneva, Lausanne, Paris and Zurich. Almost all non-musicians and amateurs were students at the Universities of Geneva and Lausanne. Two exceptions occurred, one non-musician was a young university professor, and the youngest amateur was still in secondary education (final year). Inclusion/exclusion criteria were the following: non-musicians should not have received any extracurricular musical education and never have practised a musical instrument; piano practice of amateurs should never have exceeded $10 \mathrm{~h}$ training per week and their musical practice should have been continuous from childhood until the moment of testing; the latter condition of course also applied to the experts.

Musicians (amateurs and experts) should have started their piano practice at the age of ten at the latest. Amateurs started studying the piano at $7.0 \pm 1.4$ years, experts at $6.2 \pm 1.9$ years; age at beginning of practice was not significantly different between the two musician groups $\left(t_{38}=1.5, p=0.19\right)$. By means of a questionnaire we assessed individual training intensity in different age brackets (in years: 6-8, 8-10, 10-12, 12-14, 14-16, 16-18, $18-25)$. For the first two periods $(6-8,8-10)$ we found no significant differences in reported training intensity between amateurs and experts, whereas the subsequent periods revealed a pattern of consistently increasing training lag of amateurs compared to experts (Table 1).

All subjects also participated in an fMRI study reported in Oechslin et al. (2012). This in-scanner behavioural task is described at the end of this Methods session in "Behavioural measures". The results from this task allowed verifying the progressive increase in musical expertise of the groups.

Data acquisition and analysis

We recorded a T1-weighted 3-D gradient-echo structural image for each individual (MPRAGE, TE $=2.27 \mathrm{~ms}$, $\mathrm{TR}=1900 \mathrm{~ms}$, flip angle $=9^{\circ}, \mathrm{FOV}=256 \times 256 \mathrm{~mm}$,
Table 1 Training intensities of amateur and expert pianists reported in mean $( \pm \mathrm{SD})$ number of hours/week $(\mathrm{h} / \mathrm{w})$ within consecutive age brackets

\begin{tabular}{llll}
\hline Training intensity & & & \\
\hline Age bracket & A $(\mathrm{h} / \mathrm{w})$ & $\mathrm{E}(\mathrm{h} / \mathrm{w})$ & $t$ values \\
\hline $6-8$ & $3.0( \pm 1.9)$ & $3.1( \pm 1.7)$ & $t_{26}: 1.1$ \\
$8-10$ & $3.5( \pm 0.5)$ & $4.2( \pm 0.5)$ & $t_{38}: 1.1$ \\
$10-12$ & $4.0( \pm 2.3)$ & $6.5( \pm 4.3)$ & $t_{38}: 2.7 *$ \\
$12-14$ & $4.7( \pm 2.6)$ & $9.0( \pm 5.3)$ & $t_{38}: 3.3^{* *}$ \\
$14-16$ & $5.3( \pm 3.2)$ & $14.8( \pm 7.7)$ & $t_{38}: 5.1^{* * *}$ \\
$16-18$ & $4.7( \pm 2.2)$ & $19.9( \pm 9.3)$ & $t_{38}: 7.1^{* * *}$ \\
$18-25$ & $4.8( \pm 2.6)$ & $30.7( \pm 8.5)$ & $t_{38}: 12.4^{* * *}$ \\
\hline
\end{tabular}

Groups were not yet complete in the first age bracket

Differences were assessed by $t$ tests (two-tailed) for each bracket, asterisks indicate level of significance: $* p<0.05$, ** $p<0.01$, *** $p<0.001$

slice thickness $=1 \mathrm{~mm}$, inversion time $(\mathrm{TI})=900 \mathrm{~ms}$, voxel size $=1 \times 1 \times 1 \mathrm{~mm}$, intensity correction with Prescan Normalize) on a 3-tesla MRI scanner (Siemens TIM-TRIO, Erlangen, Germany). Data analysis was performed with statistical parametric mapping software (SPM8, Welcome Department of Imaging Neuroscience, London, UK).

The pre-processing pipeline for the voxel-based morphometry (VBM) analyses comprised the following steps. Brain tissue was segmented into grey matter (GM), white matter (WM) and cerebrospinal fluid (CSF) using the new segment procedure of SPM8. This tool represents an improved version of the default unified segmentation method (Ashburner and Friston 2005). Images were transformed nonlinearly to standard MNI space using the diffeomorphic registration algorithm (DARTEL) implemented in SPM8 (Ashburner 2007). GM probability maps were then "modulated" by the Jacobian determinants of the deformations to account for local compression and expansion due to linear and non-linear transformations (Good et al. 2001). In a final step, modulated GM probability maps were smoothed with an isotropic Gaussian kernel of 8-mm full width at half maximum (FWHM).

Based on behavioural results from exactly the same participants in a musical expectation violation detection test (Oechslin et al. 2012) in which amateurs demonstrated an intermediate level of performance between experts and non-musicians, we modelled musical expertise as a threelevel regressor $\quad$ (non-musicians $=1 ; \quad$ amateurs $=2$; experts $=3$ ) to study possible linear relationships between musical expertise and grey matter density (GMD). We corrected for differences in total intra cranial volume (sum of GM, WM and CSF, computed with the SPM extension easy volume) and age, integrating covariate vectors for these two variables into the regression model (Ridgway 
et al. 2008). In order to include only relatively homogeneous voxels, and to exclude possible edge effects around the grey and white matter borders, we excluded all voxels with GMD values below 0.2 of the maximum value. We then performed a linear regression analysis (Analysis I) on GMD with the expertise vector as predictor and studied positive (GMD increase) and negative (GMD decrease) effects of expertise.

Subsequently, we extracted mean first eigenvariates (EVs) for all brain clusters exhibiting significant positive or negative effects of expertise (outcome of Analysis I) for each individual. The extraction of these EVs from the GMD values of the significant clusters was computed independently, and not adjusted to the linear regression model of Analysis I. EVs were extracted with SPM8 by means of singular value decomposition; they provide one GMD value for each cluster for each individual. SPM extracts the EVs for a cluster, rather than the mean values, because the former are more robust and resistant to heterogeneity of response distribution within all voxels of a cluster. We used these EVs to determine, which of these brain clusters were the best predictors for two different behavioural measures, via multiple linear regression analyses (Analysis II) separately for positive and negative effects of expertise. The behavioural task that yielded both behavioural measures is described below. A multiple regression analysis determines which predictors among several best predict a certain variable and thus enables the ranking of predictors according to their relative predictive influence on that variable.

\section{Behavioural measures}

We used two behavioural measures as dependent variables for Analysis II collected from the same participant group (Oechslin et al. 2012) during an event-related sparse temporal sampling ("silent") fMRI protocol, optimal for the presentation of auditory stimuli (Hall et al. 1999). Thirty original polyphone expressive musical stimuli ${ }^{1}$ were presented in pseudo-randomized order at three levels of syntactical transgression at musical closure: regular, subtly transgressed and apparently transgressed endings. An example of a stimulus at all transgression levels is provided in the supplementary material, as well as musical scores and corresponding sound files.

Participant's appraisals consisted in expressing whether end formulas (cadences) were correct, yes or no, by means

\footnotetext{
${ }^{1}$ The compositions were electronic versions of specifically composed string quartets with a duration of approximately $10 \mathrm{~s}$. The musical pieces were prepared with the "Sibelius" software (Avid Technology, Inc.) and "Logic Pro" (Apple Inc.); instrumental timbres were implemented using the "Garritan Personal Orchestra" (Garritan).
}

of button presses. From these binary results we computed d-prime values (Macmillan and Creelman 1997) for, respectively, the appraisals of subtly $\left(\mathrm{T}^{\text {sub }}\right)$ and apparently $\left(\mathrm{T}^{\mathrm{app}}\right)$ transgressed musical end formulas (see "Results"; Table 2). The d-prime index is a statistic derived from signal detection theory (Macmillan and Creelman 1997) and provides an index of rater sensitivity: higher d-prime values indicate that the transgressed ending was better detected.

For details on the experimental task and materials, and for details on the fMRI study as well as complete results please see Oechslin et al. (2012).

\section{Results}

Behavioural results (Oechslin et al. 2012)

An ANOVA on d-prime values (see Table 2) with the factors expertise (3, between) and transgression (2, within) yielded significant main effects for expertise and transgression, as well as significant interaction between the two factors: expertise: $F_{56.2}=51.4, p<0.001$; transgression: $F_{56.1}=210, p<0.001$; expertise $\times$ transgression $F_{56.2}=$ 13.6, $p<0.001$ (data from Oechslin et al. 2012; cf. "In scanner behavioural results").

In order to verify the intermediate position of the amateurs for these behavioural results, a test consisting of two combined difference contrasts (non-musicians $=-1$, amateurs $=1$, experts $=0$ and non-musicians $=-0$, amateurs $=-1$, experts $=1)$ confirmed the intermediate position of the amateurs both for $\mathrm{T}^{\mathrm{sub}}\left(F_{56,2}=51.9, p<0.001\right)$ and $\mathrm{T}^{\mathrm{app}}$ $\left(F_{56,2}=39.2, p<0.001\right)$. Figure 1 illustrates the intermediate position of the amateurs for d-prime for $\mathrm{T}^{\mathrm{sub}}, \mathrm{T}^{\mathrm{app}}$ and for mean d-prime of both transgressions.

\section{VBM results}

Anatomical labels were assigned according to cytoarchitectonic probabilities using the SPM anatomy toolbox (Eickhoff et al. 2007).

Table 2 Mean d-prime values $( \pm \mathrm{SD})$ or rater sensitivity of nonmusicians $(\mathrm{N})$, amateurs $(\mathrm{A})$ and experts $(\mathrm{E})$ for subtle transgressions $\left(\mathrm{T}^{\mathrm{sub}}\right)$ and apparent transgressions $\left(\mathrm{T}^{\mathrm{app}}\right)$ of end-formulas in expressive music

\begin{tabular}{llll}
\hline Rater sensitivity & \multicolumn{2}{l}{ Expertise } & \\
\cline { 2 - 5 } & $\mathrm{N}$ & $\mathrm{A}$ & $\mathrm{E}$ \\
& $(n=19)$ & $(n=20)$ & $(n=20)$ \\
\hline d-prime T $^{\text {sub }}($ mean $\pm \mathrm{SD})$ & $0.4 \pm 0.7$ & $1.2 \pm 0.8$ & $3.0 \pm 0.9$ \\
$\begin{array}{l}\text { d-prime T } \\
\text { Mean d-prime } \\
\begin{array}{l}\text { Mean } \\
(\text { mean } \pm \mathrm{SD})\end{array}\end{array}$ & $1.4 \pm 1.2$ & $3.5 \pm 1.2$ & $4.2 \pm 0.6$ \\
\hline
\end{tabular}


Analysis I

The linear regression analysis (Analysis I) on GMD with expertise (non-musicians $=1$; amateurs $=2$; experts $=3$ ) as predictor, corrected for age and total intracranial volume, executed with SPM8 yielded the following results (Fig. 2).

Positive effects of expertise

A positive effect of expertise implies here a stepwise increase in GMD as a function of the three degrees of expertise and thus that GMD was highest in professional musicians or experts, intermediate in amateur musicians,

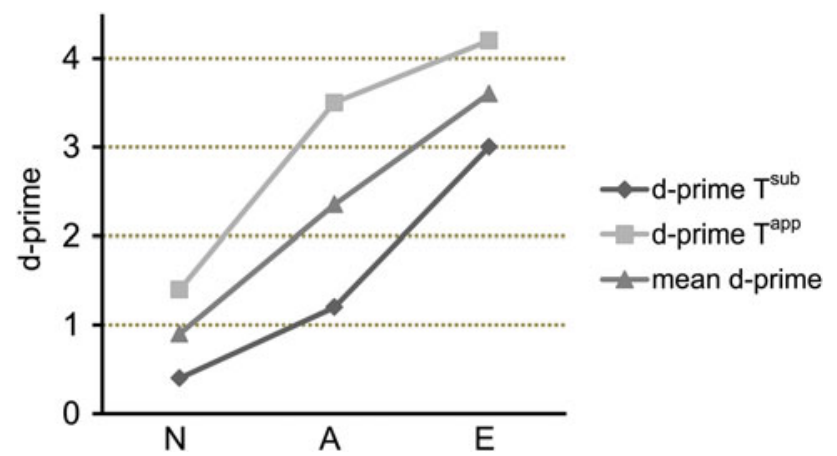

Fig. 1 Mean d-prime values for responses to end-formulas in expressive music of non-musicians (N), amateurs (A) and experts (E) for subtle transgressions ( $\left.\mathrm{T}^{\text {sub }}\right)$ in dark grey, apparent transgressions $\left(\mathrm{T}^{\mathrm{app}}\right)$ in light grey and mean d-prime for both transgressions in intermediate grey and lowest in non-musicians (red clusters in Fig. 2). All brain clusters with such significant stepwise increase are summarized in Table 3. Statistical significance was threshold at $p<0.001$ (unc.) and only clusters with a minimum of 30 voxels were retained.

At the initial threshold of $p<0.001$ (unc.), there was no evidence of enhanced GMD in the primary auditory cortex/ Heschl's gyrus. However, many important studies demonstrated grey matter volumetric increases in this area (Bermudez et al. 2009; Bermudez and Zatorre 2005; Gaser and Schlaug 2003; Schneider et al. 2002, 2005). In order to verify whether our regression model could show stepwise increase in GMD in auditory cortices as a function of expertise, we executed supplementary regression analyses, using the same three-level regressor, in two regions of interest: the left and right superior temporal gyri, now with a more liberal threshold of $p<0.005$ (unc.) and again a minimum cluster size of 30 voxels. The regions of interest were extracted using the AAL atlas (automated anatomical labelling (Tzourio-Mazoyer et al. 2002), via the Pickatlas toolbox implemented in SPM8.

Positive effects (Table 3) in cortical areas exhibited, respectively, in a small cluster in the medial posterior part of the right fusiform gyrus (peak effect), in the right mid (medial) orbital gyrus, in the left inferior frontal gyrus (this cluster extends from the pars triangularis into more medial regions in the anterior insula; see Fig. 2d.4.) and in the left inferior parietal lobule, more precisely in the grey matter of the anterior intraparietal sulcus.
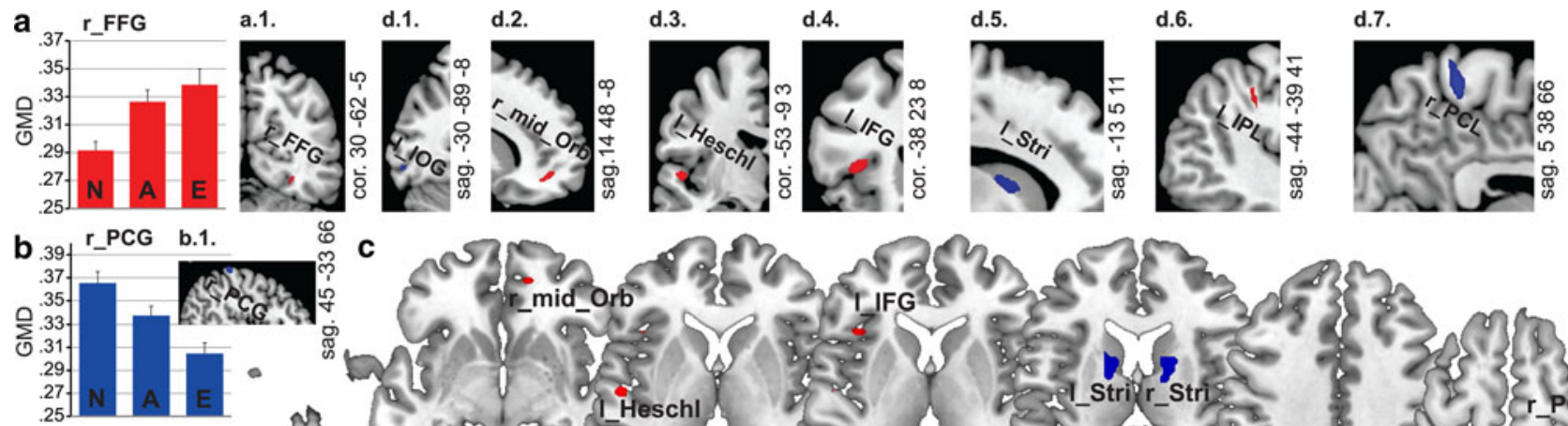

C
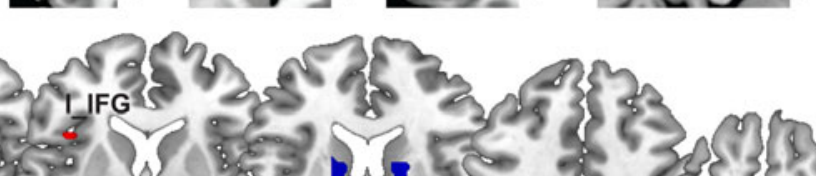

d.8.
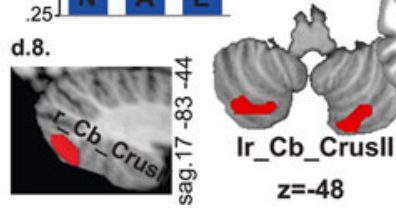

$z=-48$

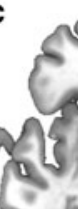

Fig. 2 Brain clusters with significant positive (red) and negative (blue) effects of expertise on GMD ( $p<0.001$, unc., $k=30$; only for 1_Heschl $p<0.005$, unc., $k=30$ ). a Positive peak effect in r_FFG, a.1., detailed view of r_FFG in coronal orientation. $\mathbf{b}$ Negative peak effect in r_PCG. b.1., detailed view of r_PCG in sagittal orientation. c All other positive and negative effects of expertise shown on axial slides at seven different $\mathrm{z}$ levels. d.1.-8. Detailed views of all other areas showing positive and negative effects of expertise. MRI

coordinates and orientations are provided vertically. Orientations: cor. coronal, sag. sagittal. Positive effects: r_FFG, right fusiform gyrus; r_mid_Orb, right mid orbital gyrus; r_Cb_CrusII, right cerebellum Crus II; 1_IFG, left inferior frontal gyrus; 1_IPL, left inferior parietal lobule; 1_Cb_CrusII, left cerebellum Crus II; 1_Heschl, left Heschl's gyrus. Negative effects: r_PCG, right postcentral gyrus; 1_PCL, left paracentral lobule; r_Stri, right striatum; 1_IOG, left inferior occipital gyrus; 1_Stri, left striatum 
Table 3 Significant clusters with increased GMD as a function of expertise, identified by a regression analysis (Analysis I) corrected for intracranial volume and age

\begin{tabular}{lcccccr}
\hline Regions & Voxels & $T_{\max }$ & $Z_{\max }$ & \multicolumn{2}{c}{ MNI coord. peak voxel } \\
\hline r_FFG & 35 & 4.05 & 3.77 & 30 & -62 & -5 \\
r_mid_Orb & 74 & 3.87 & 3.62 & 14 & 48 & -8 \\
r_Cb_CrusII & 537 & 3.83 & 3.59 & 17 & -83 & -44 \\
1_IFG & 83 & 3.82 & 3.58 & -38 & 23 & 8 \\
1_IPL & 49 & 3.66 & 3.45 & -44 & -39 & 41 \\
1_Cb_CrusII & 388 & 3.66 & 3.44 & -35 & -68 & -47 \\
1_Heschl & 81 & 3.03 & 2.90 & -53 & -9 & 3 \\
\hline
\end{tabular}

Anatomical labels (MNI coordinates of peak voxels) are provided. Expertise was defined as a 3-level regressor (non-musicians $=1$, amateurs $=2$; experts $=3$ ). Only clusters with a minimum of 30 voxels were retained

Statistical significance was threshold at $p<0.001$ (unc.). The last cluster in Heschl's gyrus was threshold at $p<0.005$ (unc.)

$r \_F F G$ right fusiform gyrus, $r \_m i d \_O r b$ right mid orbital gyrus, $r \_C b \_C r u s I I$ right cerebellum Crus II, $l_{-} I F G$ left inferior frontal gyrus, $l_{-} I P L$ left Inferior parietal lobule, $I_{-} C b_{-} C r u s I I$ left cerebellum Crus II, l_Heschl left Heschl's gyrus

Positive effects in cerebellar areas manifested in large bilateral clusters in the posterior lobe, in Crus II of Lobule VIIa.

The supplementary region of interest analysis in the left and right superior temporal gyrus yielded significant results in the left primary auditory cortex, namely in the anterior lateral area of Heschl's gyrus or Te 1.2 (Morosan et al. 2001).

Negative effects of expertise

A negative effect of expertise implies here a stepwise decrease in GMD following the three degrees of musical expertise and thus that GMD was lowest in experts, intermediate in amateur musicians, and highest in non-musicians (blue clusters in Fig. 2). All brain clusters with such significant stepwise decrease are summarized in Table 4.

Negative effects manifested, respectively, in the right postcentral gyrus (peak effect), in bilateral precuneus/paracentral lobule, left inferior occipital gyrus and bilateral striatal areas.

The peak voxel was found in the right postcentral gyrus (assigned to sensory area 1 according to Eickhoff et al. 2007). The bilateral paracentral clusters reached, in their most rostral and medial extents, the borders of the precuneus, and more anteriorly the paracentral lobules (assigned to area M1-4a according to Eickhoff et al. 2007). These regions are situated on the junction between the most anterior part of the precuneus and the primary motor cortex (M1). The precuneus and M1 are adjacent (on both sides of the central sulcus) in the most medial part of the brain.

The bilateral striatal clusters encompassed parts of the caudate nucleus, the borders of the putamen, as well as
Table 4 Significant clusters with decreased GMD as a function of expertise, identified by a regression analysis (Analysis I) corrected for intracranial volume and age

\begin{tabular}{lcccrrr}
\hline Regions & Voxels & $T_{\max }$ & $Z_{\max }$ & \multicolumn{2}{c}{ MNI coord. peak voxel } \\
\hline r_PCG & 120 & 4.92 & 4.46 & 48 & -33 & 66 \\
1_Prec/1_PCL & 432 & 4.35 & 4.02 & -5 & -41 & 66 \\
r_Prec/r_PCL & & & & 5 & -38 & 66 \\
r_Stri & 235 & 3.92 & 3.66 & 18 & 5 & 9 \\
1_IOG & 40 & 3.79 & 3.56 & -30 & -89 & -8 \\
1_Stri & 237 & 3.52 & 3.33 & -15 & 5 & 11 \\
\hline
\end{tabular}

Anatomical labels (MNI coordinates of peak voxels) are provided. Expertise was defined as a 3-level regressor (non-musicians $=1$, amateurs $=2$; experts $=3$ ). Only clusters with a minimum of 30 voxels were retained

Statistical significance was threshold at $p<0.001$ (unc.)

$r \_P C G$ right postcentral gyrus, $l \_P r e c$ left precuneus/1_PCL left paracentral lobule, $r \_S t r i$ right striatum, $I_{-} I O G$ left inferior occipital gyrus, __Stri left striatum

grey matter tissue stripes within white matter regions in between those two nuclei (see Fig. 2c, d.5.). These grey matter strands, at the origin of the name of this region, cannot be appreciated on MRI templates, but can be clearly visualized in sections of the human brain at the level of a coronal plane passing through the anterior commissure (Duvernoy 1991, pp. 226-227). All brain areas that showed positive and negative effects of expertise are depicted encompassing their peak voxels in Fig. 2c, more extensive representation of significant voxels is provided in Fig. 2d.1.-8. for all brain clusters.

\section{Analysis II}

For each participant, we extracted one mean first eigenvariate $(\mathrm{EV})$ for each brain cluster that gave rise to significant increase or decrease of GMD as a function of expertise in Analysis I. These EVs were implemented in multiple regressions analyses as predictors for behavioural results, respectively, for d-prime of $\mathrm{T}^{\text {sub }}$ (subtle syntactic transgression) and $\mathrm{T}^{\text {app }}$ (apparent syntactic transgression; see "Methods" section), separately for positive and negative effects of expertise.

Residuals were normally distributed for all regression equations. One outlier in the expert group was excluded from the analyses. The results from both multiple regression analyses for $\mathrm{T}^{\text {sub }}$ and $\mathrm{T}^{\text {app }}$ for areas manifesting positive effects of expertise are provided in Table 5. Both multiple regression equations were highly significant (top lines of Table 5), so all brain clusters together predicted the behavioural outcomes (d-prime for $\mathrm{T}^{\text {sup }}$ and $\mathrm{T}^{\mathrm{app}}$, respectively) very well. For $\mathrm{T}^{\text {sub }}$, two individual brain clusters within the model predicted the behavioural outcome 
significantly: the right mid orbital gyrus and left inferior frontal gyrus (i.e. pars triangularis and anterior insula). In these areas, increased GMD resulted in significantly better detection of $\mathrm{T}^{\mathrm{sub}}$. For $\mathrm{T}^{\mathrm{app}}$ not one individual cluster predicted behaviour significantly; however, the right mid orbital gyrus reached marginal significance. Results from both multiple regression analyses for, respectively, $\mathrm{T}^{\text {sub }}$ and $\mathrm{T}^{\mathrm{app}}$ for areas manifesting negative effects of expertise are provided in Table 6. Again both equations were highly significant (top lines of Table 6). At the level of individual regions, only the left inferior occipital gyrus predicted the behavioural outcome of both $\mathrm{T}^{\mathrm{sub}}$ and $\mathrm{T}^{\mathrm{app}}$. For this area, less GMD resulted in better performance.

\section{Discussion}

The key finding of this study resides in the observed functional dissimilarity between areas showing increase

Table 5 Results of two multiple regression analyses with as predictors all significant brain clusters with positive effect of expertise (outcome Analysis I, cf. Table 3) for (a) dependent variable d-prime $\mathrm{T}^{\text {sub }}$ (subtle transgression) and (b) dependent variable d-prime of $\mathrm{T}^{\text {app }}$ (apparent transgression)

\begin{tabular}{lclc}
\hline Predictor & Beta & SEM (beta) & $t(50)$ \\
\hline \multicolumn{2}{l}{ (a) Regression summary for dependent variable $\mathrm{T}^{\text {sub }}$; } \\
\multicolumn{2}{l}{$F_{7,50}=5.12^{* * *} ; R^{2}=0.42$} \\
r_FFG & 0.24 & 0.12 & 1.94 \\
r_mid_Orb & $\mathbf{0 . 2 8}$ & $\mathbf{0 . 1 1}$ & $\mathbf{2 . 4 6}$ \\
r_Cb_CrusII & 0.18 & 0.17 & 1.05 \\
l_IFG & $\mathbf{0 . 2 8}$ & $\mathbf{0 . 1 2}$ & $\mathbf{2 . 3 2}$ \\
1_IPL & 0.14 & 0.12 & 1.19 \\
1_Cb_CrusII & -0.07 & 0.18 & -0.39 \\
1_Heschl & 0.16 & 0.12 & 1.34
\end{tabular}

(b) Regression summary for dependent variable $\mathrm{T}^{\mathrm{app}} ; F_{7,50}=3.80^{* *}$; $R^{2}=0.35$

$\begin{array}{llll}\text { r_FFG } & 0.16 & 0.13 & 1.27 \\ \text { r_mid_Orb } & 0.23 & 0.12 & 1.91^{\circ} \\ \text { r_Cb_CrusII } & 0.22 & 0.18 & 1.20 \\ \text { 1_IFG } & 0.17 & 0.13 & 1.34 \\ \text { 1_IPL } & 0.18 & 0.12 & 1.41 \\ \text { 1_Cb_CrusII } & 0.03 & 0.19 & 0.16 \\ \text { 1_Heschl } & 0.11 & 0.13 & 0.85\end{array}$

In the top panels the results for the full regression equations are reported. Below beta coefficients, standard error of mean (SEM) for beta coefficients and corresponding $t$ values for the individual predictors are reported

Dependent variable $\mathrm{T}^{\text {sub }}$ d-prime subtle transgression; SEM standard error of mean

Significant results are plotted in bold font

Asterisks indicate level of significance: $* p<0.05$; ** $p<0.01$; *** $p<0.001,{ }^{\circ} p<0.07$
Table 6 Results of two multiple regression analyses with as predictors all significant brain clusters with negative effect of expertise (outcome Analysis I, cf. Table 4) for (a) dependent variable d-prime $\mathrm{T}^{\text {sub }}$ (subtle transgression) and (b) dependent variable d-prime of $\mathrm{T}^{\mathrm{app}}$ (apparent transgression)

\begin{tabular}{llll}
\hline Predictor & Beta & SEM (beta) & $t(53)$ \\
\hline
\end{tabular}

(a) Regression summary for dependent variable $\mathrm{T}^{\text {sub; }}$;

$F_{5,52}=6.61^{* * *} ; R^{2}=0.39$

$\begin{array}{llll}\text { r_PCG } & -0.16 & 0.13 & -1.23 \\ \text { lr_Prec/PCL } & -0.10 & 0.13 & -0.72 \\ \text { r_Stri } & -0.19 & 0.21 & -0.90 \\ \text { I_OG } & \mathbf{- 0 . 3 7} & \mathbf{0 . 1 2} & \mathbf{- 3 . 1 7 * *} \\ \text { 1_Stri } & -0.13 & 0.22 & -0.62\end{array}$

(b) Regression summary for dependent variable $\mathrm{T}^{\text {app}}$; $F_{5,52}=8.14 * * * ; R^{2}=0.44$

$\begin{array}{llll}\text { r_PCG } & -0.19 & 0.13 & -1.52 \\ \text { lr_Prec } & -0.16 & 0.13 & -1.30 \\ \text { rStri } & -0.07 & 0.21 & -0.35 \\ \text { I_IOG } & \mathbf{- 0 . 4 5} & \mathbf{0 . 1 1} & \mathbf{- 4 . 0 0 * * *} \\ \text { 1_Stri } & -0.13 & 0.21 & -0.63\end{array}$

In the top panels the results for the full regression equations are reported. Below beta coefficients, standard error of mean (SEM) for beta coefficients and corresponding $t$ values for the individual predictors are reported

Dependent variable $\mathrm{T}^{\mathrm{sub}}$ d-prime subtle transgression, SEM standard error of mean

Significant results are plotted in bold font

Asterisks indicate level of significance: $* p<0.05$, ** $p<0.01$, *** $p<0.001$

versus decrease of grey matter density (GMD) as a function of musical expertise. Progressive increase of GMD as a consequence of expertise manifested in several cortical areas known to be involved in higher-order cognitive processing, primary auditory processing and in bilateral posterior cerebellar areas also implicated in cognitive behaviour. Increase in GMD as a function of musical expertise in the fusiform gyrus and in posterior cerebellar areas has not been evidenced before.

In contrast, decreases were found principally in sensorimotor function related areas. Reduction of GMD exhibited in perirolandic cortical areas and subcortical areas in the striatum, possibly induced by ruling out of non-pertinent movement and proprioceptive feedback relative to piano performance, resulting in greater efficiency and automation, thus increasing virtuosity. Such cortical and subcortical sensorimotor function related GMD reduction adds a new perspective on cerebral reorganisation with increasing motor skill.

We suggest that these findings are the consequence of our well-defined group categorizations that allowed observing progressive changes of GMD with stepwise increasing expertise as a consequence of specific training. 
The homogeneity of all groups used here and more specifically the similarity of training experiences shared by the two pianist groups may have induced the revelation of more refined differences. Diverse training experiences might have masked these findings.

\section{Analysis I}

Positive effects of expertise

The peak effect was found in the right fusiform gyrus (FFG), known for its implication in symbolic processing through visual form recognition (Koutstaal et al. 2001). Lesion studies (Leff et al. 2001) as well as functional imaging (Price and Mechelli 2005) evidenced that the left posterior FFG plays an important role in reading. Primetarget pairs of orthographically related words produced a neural priming effect in the left FFG that decreased if the prime-target pairs were semantically related (Price and Mechelli 2005). This result suggests that the FFG not just stores visual word forms but rather acts as an interface between visual form information and higher-order stimulus characteristics such as associated sound and meaning (Devlin et al. 2006). This function is not specific to text reading, but may be engaged in the processing of any meaningful visual stimulus (Devlin et al. 2006), and prone to plastic changes. Musically untrained adults who learned to read music and play the piano over a period of 3 months showed learning-related functional changes in the fusiform gyrus (Stewart 2005). Musicians trained from early childhood relied more on the left posterior fusiform gyrus for mathematical processing (addition and subtraction of fractions) than non-musicians (Schmithorst and Holland 2004). The fact that we found right-sided increase in GMD can be explained by the fact that the right cerebral hemisphere may retain more specific visual form information than the left, which may store more abstract lexicalsemantic representations (Koutstaal et al. 2001). A music expert directly links music notation to sound (Behmer and Jantzen 2011). In conclusion, we suggest that the increase in GMD in the right fusiform area with increasing expertise may reflect the acquisition of abstract musical score representations and their sound associations through progressive training. Among instrumentalists' scores, those of pianists are among the most complex.

The second cortical effect manifested in an area in the right mid orbital gyrus. No consensus seems to exist concerning the specific functions of this area. It is reported to be involved in self-referential judgement (Denny et al. 2012), in cognitive control of emotion (Ochsner et al. 2009), in metamemory (prediction of memory success, Do Lam et al. 2012) and in tracking of tonality in western tonal music (Janata et al. 2002a). It is also presumed to be part of the default network (Kim 2012) that is active during most internally oriented mental activities. We would like to adopt the interpretation by Petr Janata (Janata et al. 2002a; Janata 2005) concerning the function of this area as a "nexus of cognitive, affective and mnesic processing", with a specific sensitivity for the tracking of changes in tonality in western tonal music. That such an area develops its GMD with intensive musical training seems plausible, specifically in pianists that play polyphonic music all the time. Analysis II underpinned this presumption, as GMD in this area best predicted the detection of syntactical transgressions in tonal music.

Another important cortical effect was increased GMD in the left inferior frontal gyrus in a cluster extending from the pars triangularis into the anterior insula. Possible explanations for GMD enhancement in this area are twofold. In the first place both pars triangularis and anterior insula are well known for their role in syntactic processing of language and music (Friederici 2002; Koelsch et al. 2001; Nan and Friederici 2012; Tillmann et al. 2006). In analogy, grey matter volume increase in Broca's area (pars opercularis) could be evidenced via VBM in professional male orchestra musicians (Sluming et al. 2002). Secondly, increase of grey matter volume in these regions could also reflect enhanced general cognitive function in musicians, encompassing processes such as top-down attention and working memory (Janata et al. 2002b; Schulze et al. 2011). In conjunction with this, anterior insula activation can express stimulus driven perceptual demand (Sterzer and Kleinschmidt 2010). In a visual 3-back working memory task with letters (Oechslin et al. 2012), in which the same participant group as in the current experiment participated, the expert musicians outperformed the two other groups. This is not surprising, as attentional and working memory loads are high during musical performance. To summarize, increased GMD in the inferior frontal cortex with expertise may thus represent experience-driven brain adaptation allowing musicians to optimally retain and interpret complex auditory stimuli, in highly demanding attentional contexts such as on stage musical performance.

Moreover, in our associated fMRI study, (Oechslin et al. 2012) bilateral anterior insulae (ant_INS) were activated differentially as a function of expertise and degree of syntactical transgression (interaction effect expertise $\mathrm{x}$ transgression). In Fig. 3, the overlap of these functional and the here analyzed structural results in the left hemisphere are shown. In the fMRI study, subtle transgressions $\left(\mathrm{T}^{\text {sub }}\right)$ activated the ant_INS more strongly with increasing expertise; apparent transgressions $\left(\mathrm{T}^{\mathrm{app}}\right)$ induced the opposite effect. For details on the contrasts used in this fMRI study we refer to the original communication (Oechslin et al. 2012). This result suggests that local 


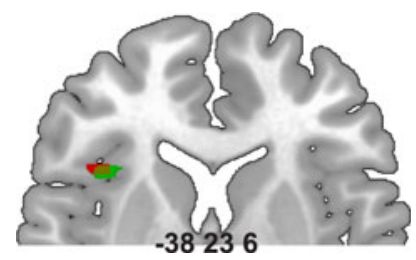

Fig. 3 Overlap of functional results (in green; interaction expertise $\times$ transgression) from Oechslin et al. (2012) and structural results of the present study (in red) in the left inferior frontal cortex. The MRI coordinate is provided

structure of grey matter as assessed by voxel-based morphometry is intimately linked to cognitive behaviour as shown before (Kanai and Rees 2011; Schneider et al. 2002).

Then, increased cortical density was observed in the left anterior intraparietal sulcus (IPS), whose functions are related to perceptual motor coordination (Simon et al. 2002). An increase in grey matter density in the left posterior IPS could be demonstrated using VBM in a longitudinal study on the plastic effects of juggling training (Draganski et al. 2004). Accordingly, although inferior parietal areas were activated during both real and imagined piano performance, the IPS exhibited significantly stronger BOLD responses during music performance compared to imagined musical behaviour (Meister et al. 2004). Furthermore, the IPS has been identified as a critical structure for note reading (Schön et al. 2002; Stewart et al. 2003). Therefore, both visuo-motor coordination (hand-keyboard) during performance and music score reading or "translating" music notation into sound may have contributed to the gradual expansion of GMD with expertise in this area.

The supplementary regression analyses in left and right superior temporal lobes demonstrated increase of GMD with expertise in the left primary auditory cortex, more precisely in the anterior lateral area of Heschl's gyrus or Te1.2 (temporal cortical area 1.2; Morosan et al. 2001; Eickhoff et al. 2007). The observation that GMD increases with musical aptitude in the primary auditory cortex confirms the literature on enhanced grey matter volume in the primary auditory cortices of musicians (Bermudez et al. 2009; Bermudez and Zatorre 2005; Gaser and Schlaug 2003; Schneider et al. 2002, 2005), and can be easily explained by musical practice. In a population of nonmusicians, amateur musicians and professional musicians, Schneider et al. (2002) evidenced bilateral increases in grey matter in Heschl's gyrus with increasing musical aptitude that could be associated with stepwise differences in strength of MEG responses to sinusoidal tones. Gaser and Schlaug (2003) and the current study found exclusively left-sided enhancement. These left-sided findings do not tally well with the literature proposing that right auditory cortex may subserve fine grained pitch (frequency) processing, specifically of musical stimuli (Zatorre et al. 2002). Several studies report bilateral or right-sided enhancement of grey matter in musicians in this area (Bermudez et al. 2009; Bermudez and Zatorre 2005; Schneider et al. 2002). It is possible that our regression model comprising two groups of pianists with different levels of expertise accounts for this left-sided observation. Instrumentalists that play percussive instruments (piano, trumpet, drums etc.), so-called "fundamental pitch listeners" showed enhanced grey matter volume in left Heschl's gyrus (Schneider et al. 2005), which is sensitive to rapid temporal processing (Zatorre et al. 2002). Gaser and Schlaug (2003) also examined exclusively keyboard players.

Bilateral cerebellar cortex exhibited massive increase of GMD with expertise in bilateral Crus II of lobule VIIa. Traditionally the cerebellum has been associated to motor function (Ito 2002). This seems a plausible explanation in the present context: increased GMD with increasing pianistic virtuosity. Nevertheless, a thorough reading of recent literature on cerebellar function rejects this point of view. Distinct loops exist between cerebellum and cortex for, respectively, cognitive and motor function. Functional neuroimaging in combination with diffusion-weighted MRI (Salmi et al. 2009) revealed that the posterior cerebellum (Crus I and II), which is connected to lateral prefrontal areas, was activated by cognitive load increase in a nonverbal auditory memory task; in contrast, the anterior cerebellum (lobules V/VI), known to be involved in sensorimotor function, was not. An analysis on resting state functional connectivity observed the same dissociation: a primary sensorimotor zone (lobules V, VI and VIII) could be distinguished from a supramodal zone (lobules VIIa, Crus I, and II). The cortical connectivity of the supramodal zone was driven by areas of frontal and parietal cortex that are not directly involved in sensorimotor functioning (O'Reilly et al. 2010). A meta-analysis (53 studies) on cerebellar function concluded that language and executive tasks activated regions of Crus I and lobule VII supposed to be engaged in prefrontal-cerebellar loops (Stoodley and Schmahmann 2009); these authors thus also suggest an anterior sensorimotor versus posterior cognitive/emotional dichotomy in the cerebellum. We conclude that the increased GMD in bilateral Crus II observed here, thus, reflects improved working memory and executive function. As stated with respect to the inferior frontal cortex increase above, attention and working memory loads are high during musical performance.

Negative effects of expertise

We observed a decrease of GMD in sensorimotor areas, in the right postcentral gyrus (sensory area 1; Eickhoff et al. 
2007), bilateral paracentral lobule (comprising the precuneus and also M1-4a; Eickhoff et al. 2007) and bilateral striatum. M1-4a represents an area of secondary motor execution that actively participates in complex sensorimotor processing; its activation depends thus on a feedback system (Nakada et al. 2000). M1-4a even responds to sensory stimulation without involving in actual motion (Wilson et al. 2004). Conversely, M1-4p is rather involved in initiation of movement, and does not respond to sensory stimulation (Terumitsu et al. 2009). While learning to play an instrument; visual, proprioceptive and auditory feedbacks are essential, but with increasing skill, these external cues are no longer necessary (Krings et al. 2000), and can even become a nuisance and hamper smooth automatized sensorimotor function. Motor skill acquisition and mastery manifest according to principles of economy: chunking complex behaviour into compact units diminishes the number of degrees of freedom. With enhanced skill, fewer neurons are recruited for the same movements, which show a decrease in movement variability, and less effort is necessary for their execution, allowing musicians to progressively dedicate their attention to artistic goals rather than sensorimotor ones (Krings et al. 2000; Jancke et al. 2000). Finally, internally generated movement deriving from a forward model gives rise to motor-induced suppression of sensory cortical feedback (Aliu et al. 2009).

The striatum also follows this principle of parsimony. In professional ballet dancers a reduction in putaminal GMD compared with control subjects could be evidenced. Granert et al. (2011) also observed that the basal ganglia deviate from the rule "the more volume, the higher the level of skill". Better timing of key stroke, characterising skill level of piano playing, could be associated with less grey matter volume in the putamen of pianists compared to healthy non-musician controls, whereas an increase of GMD manifested in pianists suffering from musician's dystonia. ${ }^{2}$ Apparently high movement skill reduces the importance of striatal movement control. Models describing this focusing of cortico-basal ganglia-thalamo-cortical loops that strengthen relevant cortical inputs while suppressing irrelevant ones have been described in the past (Mink 1996, 2003; Bar-Gad et al. 2003). Our study demonstrates a GMD decrease in the border region of the putamen, but more clearly in the caudate nucleus, as well as in their connections passing through the internal capsule. Chess experts, who also depend on quick automatized responses like expert musicians, also manifested a reduced volume in the caudate nucleus (Duan et al. 2012). This phenomenon also manifested in other board games and skills (Wan et al. 2011; Poldrack et al. 2005). In

\footnotetext{
2 Musician's dystonia or focal dystonia is a neurological disorder that affects muscle control in specific often overtrained areas.
}

accordance, we show for the first time a stepwise GMD decrease in the caudate nucleus with musical aptitude.

Finally, the cluster in the left inferior occipital area that also displayed decrease in GMD with expertise is virtually the same that was found less activated in musicians in the right hemisphere, during math processing relative to nonmusicians (Schmithorst and Holland 2004). Interestingly the same study found increased activation in the fusiform area. The authors explained the decreased activation in the inferior occipital area as a habituation effect, a practice effect shown before in the context of visuospatial working memory and stimulus repetition (Garavan et al. 2000; Koutstaal et al. 2001). We suppose that pianists, following intensive daily practice, rely on the fusiform gyrus for decoding complex musical scores and, therefore, recruit the secondary visual areas less, resulting in a decrease of GMD.

\section{Analysis II}

The first multiple regression analysis using all brain clusters with positive effects of expertise as predictors for $\mathrm{T}^{\text {sub }}$ (subtle transgression) revealed that the right mid orbital gyrus cluster best explained sensitivity (d-prime). This strengthens our hypothesis that this area is implicated in tracking of tonality (Janata et al. 2002a), a faculty that demands mnesic, cognitive and also affective processing. It is through changes of tonality that western classical composers induced affect over the centuries. This affective processing may manifest as a form of relevance detection, as is also suggested for the amygdala in certain contexts (Ousdal et al. 2008). We suggest that such relevance detectors play an important role in the dynamic context of stage performance and allow a professional to quickly adapt in incongruous musical situations due to individual or contextual errors (James et al. 2008). Second best in explaining sensitivity for $\mathrm{T}^{\mathrm{sub}}$ was the left inferior frontal gyrus cluster. This result stresses the importance of this area for syntax processing, in conjunction with our functional imaging results (Oechslin et al. 2012). The latter study evidenced increase of activation with expertise in the bilateral anterior insulae for $\mathrm{T}^{\text {sub }}$ in an area that overlaps in the left hemisphere with positive effect of expertise of the current study (see Fig. 3). Detection of these subtle transgressions increased with expertise (see Fig. 1).

The second multiple regression analysis using all brain clusters with positive effects of expertise as predictors for $\mathrm{T}^{\text {app }}$ (apparent transgression) did not reveal any significant results for individual predictors. The right mid orbital gyrus cluster best explained sensitivity (d-prime) again, but this effect was only marginally significant.

The third and fourth regression analyses using all brain clusters with negative effects of expertise as predictors for, 
respectively, $\mathrm{T}^{\mathrm{sub}}$ and $\mathrm{T}^{\mathrm{app}}$ indicated that one area present among the predictors, the left inferior occipital gyrus, predicted accuracy significantly in a negative way: less GMD in this area correlated with higher d-prime values. This intriguing finding may be related to the increase of GMD in the right fusiform area with expertise. Complex score reading, essential for pianists, may progressively induce specific pattern recognition with increasing expertise in the right fusiform gyrus, consequently reducing involvement of more basic visual areas. Therefore, the reduction of GMD in occipital areas may indirectly reflect enhanced pattern recognition, subserving score reading but possibly also musical syntactical processing, operated in the fusiform gyrus.

\section{Comparison to the literature}

A similar study also using VBM showed different, but equally plausible results (Gaser and Schlaug 2003). These authors also acquired rapid acquisition gradient echo scans, with the same voxel sizes, although on a $1.5 \mathrm{~T}$ scanner versus a $3 \mathrm{~T}$ scanner here. They also applied VBM; although not using Dartel tools, or New Segment that did not exist at the time, and used a larger smoothing kernel before applying statistics (12 FWHM vs. 8 FWHM here). They also investigated non-musicians, amateur and expert pianists, with groups of the same size, but only male participants. Statistical analyses were similar (three level expertise regressor), although different statistical thresholds and cluster sizes were applied. First, GMD increase in the cerebellum exhibited in anterior sensorimotor regions, whereas we found increase in posterior cerebellar areas linked to executive function. These differences in cerebellar areas may be explained by the presence of $50 \%$ of women in our sample. Only in men, not in women, an increase of relative and absolute cerebellar volume has been observed between musicians and non-musicians (Schlaug 2001; Hutchinson et al. 2003). Therefore, the presence of women in our sample may have masked possible effects of musicianship in motor function related areas of the cerebellum as found in men by Gaser and Schlaug (2003). Like in the current study, left Heschl's gyrus, left inferior frontal gyrus and right medial frontal gyrus showed enhanced GMD with increased musical expertise. However, Gaser and Schlaug (2003) found strong GMD increase in perirolandic areas, whereas we found decrease. The explanation resides, in our opinion, in the choice of the participants, namely our highly controlled and unique inclusion/exclusion criteria that shed a new light on training-induced grey matter plasticity. We could verify by means of behavioural results that the amateur group was well situated between non-musicians and experts thus legitimating a linear model. In the above- mentioned study, the group of professional musicians practiced at least $1 \mathrm{~h}$ a day; in our expert group the mean was almost $5 \mathrm{~h}$ per day. Our amateurs practiced almost an hour per day (see Table 1). Therefore, the degree of automaticity and skill of motor performance in our musician groups was enhanced in all likelihood. Intensity of practice can be associated with degree of automaticity and associated with decreased activation in sensorimotor areas (Poldrack et al. 2005). Practice strongly influences longterm retention of motor skills (Dayan and Cohen 2011). A longitudinal study with a group of established expert pianists indicated that daily practice time exceeding $3.75 \mathrm{~h}$ or more induced an improvement in a specific motor skill. The authors concluded that even in established expert pianists, maintenance of motor skills is strongly influenced by current practice quantity (Jabusch et al. 2009). This observation can be generalized; amount of deliberate practice is closely related to performance level in musicians (Ericsson et al. 1993; Sloboda et al. 1996), chess players (Charness et al. 1996) and athletes (Starkes et al. 1996).

\section{Limitations}

A limitation of our study is that the macroscopic level of our analyses prevents us from drawing any conclusions on the nature of the underlying microscopic mechanisms. Moreover, the cross-sectional design prevents us from discriminating learning effects from genetic or epigenetic factors (Zatorre et al. 2012). Only longitudinal studies beginning in childhood can distinguish nature and nurture in a musician population.

Finally, our findings are restricted to the population studied here, namely classically schooled pianists.

\section{Conclusion}

The brain generates behaviour, and is in turn modified by the behaviour it produces (Pascual-Leone 2001). The present study yielded intriguing functional dissimilarity between areas exhibiting increase versus decrease of grey matter with musical expertise. A network of higher-order cognitive-function related areas increased in volume, whereas a network of sensorimotor function related areas showed decrease. Apparently, more resources are available for higher-order music processing with increasing expertise; in contrast the development of motor skill is accompanied by progressive ruling out of non-pertinent movement and sensory feedback relative to piano performance, resulting in greater efficiency and automation, thus increasing virtuosity. 
We also established links between brain structure and function in our participants. In the first place GMD in the right mid orbital gyrus and the left inferior frontal gyrus (pars triangularis and anterior insula) significantly predicted accuracy in detecting fine-grained incongruities in tonal music. Second, an area in the inferior frontal cortex with increased GMD as a function of musical expertise overlapped anatomically with an activation cluster of a preceding functional study (Oechslin et al. 2012), in which the same participants detected musical incongruities. These results suggest that local structure of grey matter as assessed by voxel-based morphometry is intimately linked to cognitive behaviour.

Acknowledgments We would like to thank Andres Posada, Alexis Hervais Adelman and Sebastian Rieger for assisting in MR data acquisition and help with fMRI setup, and Julien Chanal and Olivier Renaud for advice on statistical data analysis. Finally we thank Alexis Hervais Adelman once more for assistance on analyses, and precious comments on the manuscript.

\section{References}

Aliu SO, Houde JF, Nagarajan SS (2009) Motor-induced suppression of the auditory cortex. J Cogn Neurosci 21(4):791-802. doi: 10.1162 /jocn.2009.21055

Ashburner J (2007) A fast diffeomorphic image registration algorithm. Neuroimage 38(1):95-113. doi:10.1016/j.neuroimage. 2007.07.007

Ashburner J, Friston KJ (2005) Unified segmentation. Neuroimage 26(3):839-851. doi:10.1016/j.neuroimage.2005.02.018

Bar-Gad I, Morris G, Bergman H (2003) Information processing, dimensionality reduction and reinforcement learning in the basal ganglia. Prog Neurobiol 71(6):439-473. doi:10.1016/. j.pneurobio.2003.12.001

Behmer LP Jr, Jantzen KJ (2011) Reading sheet music facilitates sensorimotor mu-desynchronization in musicians. Clin Neurophysiol 122(7):1342-1347. doi:10.1016/j.clinph.2010.12.035

Bermudez P, Zatorre RJ (2005) Differences in gray matter between musicians and nonmusicians. Ann N Y Acad Sci 1060:395-399

Bermudez P, Lerch JP, Evans AC, Zatorre RJ (2009) Neuroanatomical correlates of musicianship as revealed by cortical thickness and voxel-based morphometry. Cereb Cortex 19(7):1583-1596. doi:10.1093/cercor/bhn196

Bialystok E, Depape AM (2009) Musical expertise, bilingualism, and executive functioning. J Exp Psychol Hum Percept Perform 35(2):565-574. doi:10.1037/a0012735

Blakemore SJ, Frith U (2005) The learning brain: lessons for education: a precis. Dev Sci 8(6):459-465. doi:10.1111/j.14677687.2005.00434.x

Chan AS, Ho YC, Cheung MC (1998) Music training improves verbal memory. Nature 396(6707):128. doi:10.1038/24075

Charness N, Krampe R, Mayr U (1996) The role of practice and coaching in entrepreneurial skill domains: an international comparison of life-span chess skill acquisition. In: Ericsson KA (ed) The road to excellence: the acquisition of expert performance in the arts and sciences, sports, and games. Lawrence Erlbaum Associates, Mahwah, pp 51-80

Dayan E, Cohen LG (2011) Neuroplasticity subserving motor skill learning. Neuron 72(3):443-454. doi:10.1016/j.neuron.2011. 10.008
Denny BT, Kober H, Wager TD, Ochsner KN (2012) A meta-analysis of functional neuroimaging studies of self- and other judgments reveals a spatial gradient for mentalizing in medial prefrontal cortex. J Cogn Neurosci. doi:10.1162/jocn_a_00233

Devlin JT, Jamison HL, Gonnerman LM, Matthews PM (2006) The role of the posterior fusiform gyrus in reading. J Cogn Neurosci 18(6):911-922. doi:10.1162/jocn.2006.18.6.911

Do Lam ATA, Axmacher N, Fell J, Staresina BP, Gauggel S, Wagner $\mathrm{T}$, Olligs J, Weis S (2012) Monitoring the mind: the neurocognitive correlates of metamemory. PLoS One 7(1):e30009. doi: 10.1371/journal.pone.0030009

Draganski B, Gaser C, Busch V, Schuierer G, Bogdahn U, May A (2004) Neuroplasticity: changes in grey matter induced by training. Nature 427(6972):311-312. doi:10.1038/427311a

Duan X, He S, Liao W, Liang D, Qiu L, Wei L, Li Y, Liu C, Gong Q, Chen H (2012) Reduced caudate volume and enhanced striatalDMN integration in chess experts. NeuroImage 60(2):12801286. doi:10.1016/j.neuroimage.2012.01.047

Duvernoy HM (1991) The human brain: surface, three-dimensional sectional anatomy, and MRI. Springer, New York

Eickhoff SB, Paus T, Caspers S, Grosbras MH, Evans AC, Zilles K, Amunts K (2007) Assignment of functional activations to probabilistic cytoarchitectonic areas revisited. Neuroimage 36(3):511-521. doi:10.1016/j.neuroimage.2007.03.060

Ericsson KA, Krampe RT, Tesch-Römer C (1993) The role of deliberate practice in the acquisition of expert performance. Psychol Rev 100(3):363-406. doi:10.1037/0033-295x.100.3.363

Friederici AD (2002) Towards a neural basis of auditory sentence processing. Trends Cogn Sci 6(2):78-84

Garavan H, Kelley D, Rosen A, Rao SM, Stein EA (2000) Practicerelated functional activation changes in a working memory task. Microsc Res Tech 51(1):54-63. doi:10.1002/1097-0029 (20001001)51:1<54::AID-JEMT6>3.0.CO;2-J

Gaser C, Schlaug G (2003) Brain structures differ between musicians and non-musicians. J Neurosci 23(27):9240-9245

George EM, Coch D (2011) Music training and working memory: an ERP study. Neuropsychologia 49(5):1083-1094. doi:10.1016/ j.neuropsychologia.2011.02.001

Good CD, Johnsrude IS, Ashburner J, Henson RN, Friston KJ, Frackowiak RS (2001) A voxel-based morphometric study of ageing in 465 normal adult human brains. Neuroimage 14(1 Pt 1):21-36. doi:10.1006/nimg.2001.0786

Granert O, Peller M, Jabusch HC, Altenmuller E, Siebner HR (2011) Sensorimotor skills and focal dystonia are linked to putaminal grey-matter volume in pianists. J Neurol Neurosurg Psychiatry 82(11):1225-1231. doi:10.1136/jnnp.2011.245811

Hall DA, Haggard MP, Akeroyd MA, Palmer AR, Summerfield AQ, Elliott MR, Gurney EM, Bowtell RW (1999) "Sparse" temporal sampling in auditory fMRI. Hum Brain Mapp 7(3):213-223

Hanggi J, Koeneke S, Bezzola L, Jancke L (2010) Structural neuroplasticity in the sensorimotor network of professional female ballet dancers. Hum Brain Mapp 31(8):1196-1206. doi: 10.1002/hbm.20928

Hutchinson S, Lee LH, Gaab N, Schlaug G (2003) Cerebellar volume of musicians. Cereb Cortex 13(9):943-949

Hyde KL, Lerch J, Norton A, Forgeard M, Winner E, Evans AC, Schlaug G (2009) Musical training shapes structural brain development. J Neurosci 29(10):3019-3025. doi:10.1523/ JNEUROSCI.5118-08.2009

Ito $M$ (2002) Historical review of the significance of the cerebellum and the role of Purkinje cells in motor learning. Ann N Y Acad Sci 978:273-288

Jabusch HC, Alpers H, Kopiez R, Vauth H, Altenmuller E (2009) The influence of practice on the development of motor skills in pianists: a longitudinal study in a selected motor task. Hum Mov Sci 28(1):74-84. doi:10.1016/j.humov.2008.08.001 
James CE, Britz J, Vuilleumier P, Hauert CA, Michel CM (2008) Early neuronal responses in right limbic structures mediate harmony incongruity processing in musical experts. Neuroimage 42(4):1597-1608. doi:10.1016/j.neuroimage.2008.06.025

Janata P (2005) Brain networks that track musical structure. Ann N Y Acad Sci 1060:111-124

Janata P, Birk JL, Van Horn JD, Leman M, Tillmann B, Bharucha JJ (2002a) The cortical topography of tonal structures underlying Western music. Science 298(5601):2167-2170

Janata P, Tillmann B, Bharucha JJ (2002b) Listening to polyphonic music recruits domain-general attention and working memory circuits. Cogn Affect Behav Neurosci 2(2):121-140

Jancke L (2009) The plastic human brain. Restor Neurol Neurosci 27(5):521-538. doi:10.3233/RNN-2009-0519

Jancke L, Shah NJ, Peters M (2000) Cortical activations in primary and secondary motor areas for complex bimanual movements in professional pianists. Brain Res Cogn Brain Res 10(1-2): $177-183$

Kanai R, Rees G (2011) The structural basis of inter-individual differences in human behaviour and cognition. Nat Rev Neurosci 12(4):231-242. doi:10.1038/nrn3000

Kim H (2012) A dual-subsystem model of the brain's default network: self-referential processing, memory retrieval processes and autobiographical memory retrieval. Neuroimage. doi: 10.1016/j.neuroimage.2012.03.025

Koelsch S, Maess B, Gunter TC, Friederici AD (2001) Neapolitan chords activate the area of Broca. A magnetoencephalographic study. Ann N Y Acad Sci 930:420-421

Koutstaal W, Wagner AD, Rotte M, Maril A, Buckner RL, Schacter DL (2001) Perceptual specificity in visual object priming: functional magnetic resonance imaging evidence for a laterality difference in fusiform cortex. Neuropsychologia 39(2):184-199

Krings T, Topper R, Foltys H, Erberich S, Sparing R, Willmes K, Thron A (2000) Cortical activation patterns during complex motor tasks in piano players and control subjects. A functional magnetic resonance imaging study. Neurosci Lett 278(3): 189-193

Leff AP, Crewes H, Plant GT, Scott SK, Kennard C, Wise RJ (2001) The functional anatomy of single-word reading in patients with hemianopic and pure alexia. Brain 124(Pt 3):510-521

Macmillan NA, Creelman CD (1997) d'plus: a program to calculate accuracy and bias measures from detection and discrimination data. Spat Vis 11(1):141-143

Meister IG, Krings T, Foltys H, Boroojerdi B, Muller M, Topper R, Thron A (2004) Playing piano in the mind-an fMRI study on music imagery and performance in pianists. Brain Res Cogn Brain Res 19(3):219-228

Mink JW (1996) The basal ganglia: focused selection and inhibition of competing motor programs. Prog Neurobiol 50(4):381-425. doi:10.1016/s0301-0082(96)00042-1

Mink JW (2003) The basal ganglia and involuntary movements: impaired inhibition of competing motor patterns. Arch Neurol 60(10):1365-1368. doi:10.1001/archneur.60.10.1365

Moreno S, Marques C, Santos A, Santos M, Castro SL, Besson M (2009) Musical training influences linguistic abilities in 8-yearold children: more evidence for brain plasticity. Cereb Cortex 19(3):712-723. doi:10.1093/cercor/bhn120

Morosan P, Rademacher J, Schleicher A, Amunts K, Schormann T, Zilles K (2001) Human primary auditory cortex: cytoarchitectonic subdivisions and mapping into a spatial reference system. Neuroimage 13(4):684-701. doi:10.1006/nimg.2000.0715

Nakada T, Suzuki K, Fujii Y, Matsuzawa H, Kwee IL (2000) Independent component-cross correlation-sequential epoch (ICS) analysis of high field fMRI time series: direct visualization of dual representation of the primary motor cortex in human. Neurosci Res 37(3):237-244
Nan Y, Friederici AD (2012) Differential roles of right temporal cortex and Broca's area in pitch processing: evidence from music and Mandarin. Hum Brain Mapp. doi:10.1002/hbm.22046

Ochsner KN, Ray RR, Hughes B, McRae K, Cooper JC, Weber J, Gabrieli JD, Gross JJ (2009) Bottom-up and top-down processes in emotion generation: common and distinct neural mechanisms. Psychol Sci 20(11):1322-1331. doi:10.1111/j.1467-9280.2009. 02459.x

Oechslin MS, Van De Ville D, Lazeyras F, Hauert C-A, James CE (2012) Degree of musical expertise modulates higher-order brain functioning. Cereb Cortex. doi:10.1093/cercor/bhs206

Oldfield RC (1971) The assessment and analysis of handedness: the Edinburgh inventory. Neuropsychologia 9(1):97-113. doi: 10.1016/0028-3932(71)90067-4

O'Reilly JX, Beckmann CF, Tomassini V, Ramnani N, JohansenBerg H (2010) Distinct and overlapping functional zones in the cerebellum defined by resting state functional connectivity. Cereb Cortex 20(4):953-965. doi:10.1093/cercor/bhp157

Ousdal OT, Jensen J, Server A, Hariri AR, Nakstad PH, Andreassen OA (2008) The human amygdala is involved in general behavioral relevance detection: evidence from an event-related functional magnetic resonance imaging Go-NoGo task. Neuroscience 156(3):450-455

Pantev C, Herholz SC (2011) Plasticity of the human auditory cortex related to musical training. Neurosci Biobehav Rev 35(10): $2140-2154$

Pascual-Leone A (2001) The brain that plays music and is changed by it. Ann N Y Acad Sci 930:315-329

Poldrack RA, Sabb FW, Foerde K, Tom SM, Asarnow RF, Bookheimer SY, Knowlton BJ (2005) The neural correlates of motor skill automaticity. J Neurosci 25(22):5356-5364. doi: 10.1523/JNEUROSCI.3880-04.2005

Price CJ, Mechelli A (2005) Reading and reading disturbance. Curr Opin Neurobiol 15(2):231-238. doi:10.1016/j.conb.2005.03.003

Rauscher FH, Shaw GL, Levine LJ, Wright EL, Dennis WR, Newcomb RL (1997) Music training causes long-term enhancement of preschool children's spatial-temporal reasoning. Neurol Res 19(1):2-8

Raven J, Raven JC, Court JH (2003) Manual for raven's progressive matrices and vocabulary scales. section 1: general overview. San Antonio, TX: Harcourt Assessment

Ridgway GR, Henley SM, Rohrer JD, Scahill RI, Warren JD, Fox NC (2008) Ten simple rules for reporting voxel-based morphometry studies. NeuroImage 40(4):1429-1435. doi:10.1016/ j.neuroimage.2008.01.003

Salmi J, Pallesen KJ, Neuvonen T, Brattico E, Korvenoja A, Salonen O, Carlson S (2009) Cognitive and motor loops of the human cerebro-cerebellar system. J Cogn Neurosci 22(11):2663-2676. doi:10.1162/jocn.2009.21382

Schlaug G (2001) The brain of musicians. A model for functional and structural adaptation. Ann N Y Acad Sci 930:281-299

Schlaug G, Jancke L, Huang Y, Staiger JF, Steinmetz H (1995) Increased corpus callosum size in musicians. Neuropsychologia 33(8):1047-1055

Schmithorst VJ, Holland SK (2004) The effect of musical training on the neural correlates of math processing: a functional magnetic resonance imaging study in humans. Neurosci Lett 354(3): 193-196

Schneider P, Scherg M, Dosch HG, Specht HJ, Gutschalk A, Rupp A (2002) Morphology of Heschl's gyrus reflects enhanced activation in the auditory cortex of musicians. Nat Neurosci 5(7): 688-694

Schneider P, Sluming V, Roberts N, Scherg M, Goebel R, Specht HJ, Dosch HG, Bleeck S, Stippich C, Rupp A (2005) Structural and functional asymmetry of lateral Heschl's gyrus reflects pitch perception preference. Nat Neurosci 8(9):1241-1247 
Schön D, Anton JL, Roth M, Besson M (2002) An fMRI study of music sight-reading. NeuroReport 13(17):2285-2289. doi: 10.1097/01.wnr.0000044224.79663.f5

Schulze K, Zysset S, Mueller K, Friederici AD, Koelsch S (2011) Neuroarchitecture of verbal and tonal working memory in nonmusicians and musicians. Hum Brain Mapp 32(5):771-783. doi: $10.1002 / \mathrm{hbm} .21060$

Simon O, Mangin JF, Cohen L, Le Bihan D, Dehaene S (2002) Topographical layout of hand, eye, calculation, and languagerelated areas in the human parietal lobe. Neuron 33(3):475-487

Sloboda JA, Davidson JW, Howe MJA, Moore DG (1996) The role of practice in the development of performing musicians. Br J Psychol 87(2):287-309. doi:10.1111/j.2044-8295.1996.tb02591.x

Sluming V, Barrick T, Howard M, Cezayirli E, Mayes A, Roberts N (2002) Voxel-based morphometry reveals increased gray matter density in Broca's area in male symphony orchestra musicians. Neuroimage 17(3):1613-1622

Sluming V, Brooks J, Howard M, Downes JJ, Roberts N (2007) Broca's area supports enhanced visuospatial cognition in orchestral musicians. J Neurosci 27(14):3799-3806. doi:10.1523/ JNEUROSCI.0147-07.2007

Starkes JL, Deakin JM, Allard F, Hodges NJ, Hayes A (1996) Deliberate practice in sports: what is it anyway? In: Ericsson KA (ed) The road to excellence: the acquisition of expert performance in the arts and sciences, sports, and games. Lawrence Erlbaum Associates, Mahwah, pp 81-106

Sterzer P, Kleinschmidt A (2010) Anterior insula activations in perceptual paradigms: often observed but barely understood. Brain Struct Funct 214(5-6):611-622. doi:10.1007/s00429-010$0252-2$

Stewart L (2005) A neurocognitive approach to music reading. Ann N Y Acad Sci 1:377-386. doi:10.1196/annals.1360.032

Stewart L, Henson R, Kampe K, Walsh V, Turner R, Frith U (2003) Brain changes after learning to read and play music. NeuroImage 20(1):71-83

Stoodley CJ, Schmahmann JD (2009) Functional topography in the human cerebellum: a meta-analysis of neuroimaging studies.
NeuroImage 44(2):489-501. doi:10.1016/j.neuroimage.2008.08. 039

Terumitsu M, Ikeda K, Kwee IL, Nakada T (2009) Participation of primary motor cortex area $4 \mathrm{a}$ in complex sensory processing: 3.0-T fMRI study. NeuroReport 20(7):679-683. doi:10.1097/ WNR.0b013e32832a1820

Tillmann B, Koelsch S, Escoffier N, Bigand E, Lalitte P, Friederici $\mathrm{AD}$, von Cramon DY (2006) Cognitive priming in sung and instrumental music: activation of inferior frontal cortex. Neuroimage 31(4):1771-1782. doi:10.1016/j.neuroimage.2006.02.028

Tzourio-Mazoyer N, Landeau B, Papathanassiou D, Crivello F, Etard O, Delcroix N, Mazoyer B, Joliot M (2002) Automated anatomical labeling of activations in SPM using a macroscopic anatomical parcellation of the MNI MRI single-subject brain. Neuroimage 15(1):273-289. doi:10.1006/nimg.2001.0978

Wan CY, Schlaug G (2010) Music making as a tool for promoting brain plasticity across the life span. Neuroscientist 16(5): 566-577. doi:10.1177/1073858410377805

Wan X, Nakatani H, Ueno K, Asamizuya T, Cheng K, Tanaka K (2011) The neural basis of intuitive best next-move generation in board game experts. Science 331(6015):341-346. doi:10.1126/ science. 1194732

Williamon A, Valentine E, Valentine J (2002) Shifting the focus of attention between levels of musical structure. Eur J Cogn Psychol 14(4):493-520. doi:10.1080/09541440143000221

Wilson SM, Saygin AP, Sereno MI, Iacoboni M (2004) Listening to speech activates motor areas involved in speech production. Nat Neurosci 7(7):701-702. doi:10.1038/nn1263

Zatorre RJ, Belin P, Penhune VB (2002) Structure and function of auditory cortex: music and speech. Trends Cogn Sci 6(1):37-46

Zatorre RJ, Chen JL, Penhune VB (2007) When the brain plays music: auditory-motor interactions in music perception and production. Nat Rev Neurosci 8(7):547-558

Zatorre RJ, Fields RD, Johansen-Berg H (2012) Plasticity in gray and white: neuroimaging changes in brain structure during learning. Nat Neurosci 15(4):528-536. doi:10.1038/nn.3045 